\title{
Quartz Crystal Microbalance Model for Quantitatively Probing the Deformation of Adsorbed Particles at Low Surface Coverage
}

\author{
Jurriaan J. J. Gillissen, ${ }^{\dagger, \ddagger}$ Joshua A. Jackman, ${ }^{\dagger, \ddagger}$ Seyed R. Tabaei, ${ }^{\dagger, \dagger}$ Bo Kyeong Yoon, ${ }^{\dagger, \ddagger}$ \\ and Nam-Joon Cho ${ }^{*, \dagger, t, \delta(0)}$ \\ ${ }^{\dagger}$ School of Materials Science and Engineering, Nanyang Technological University, 50 Nanyang Avenue 639798, Singapore
${ }^{\ddagger}$ Centre for Biomimetic Sensor Science, Nanyang Technological University, 50 Nanyang Drive 637553, Singapore
${ }^{\S}$ School of Chemical and Biomedical Engineering, Nanyang Technological University, 62 Nanyang Drive 637459, Singapore
}

Supporting Information

\begin{abstract}
Characterizing the deformation of nanoscale, soft-matter particulates at solidliquid interfaces is a demanding task, and there are limited experimental options to perform quantitative measurements in a nonperturbative manner. Previous attempts, based on the quartz crystal microbalance (QCM) technique, focused on the high surface coverage regime and modeled the adsorbed particles as a homogeneous film, while not considering the coupling between particles and surrounding fluid and hence resulting in an underestimation of the known particle height. In this work, we develop a model for the hydrodynamic coupling between adsorbed particles and surrounding fluid in the limit of a low surface coverage, which can be used to extract shape information from QCM measurement data. We tackle this

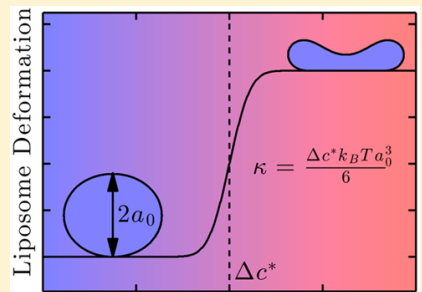

Ionic Strength Difference problem by using hydrodynamic simulations of an ellipsoidal particle on an oscillating surface.

From the simulation results, we derived a phenomenological relation between the aspect ratio $r$ of the absorbed particles and the slope and intercept of the line that fits instantaneous, overtone-dependent QCM data on $(\delta / a,-\Delta f / n)$ coordinates where $\delta$ is the viscous penetration depth, $a$ is the particle radius, $\Delta f$ is the QCM frequency shift, and $n$ is the overtone number. The model was applied to QCM measurement data pertaining to the adsorption of $34 \mathrm{~nm}$ radius, fluid-phase and gel-phase liposomes onto a titanium oxide-coated surface. The osmotic pressure across the liposomal bilayer was varied to induce shape deformation. By combining these results with a membrane bending model, we determined the membrane bending energy for the gel-phase liposomes, and the results are consistent with literature values. In summary, a phenomenological model is presented and validated in order to show for the first time that QCM experiments can quantitatively measure the deformation of adsorbed particles at low surface coverage.
\end{abstract}

M easuring the conformation of optically inaccessible (sub$100 \mathrm{~nm}$ ), soft matter adsorbates, for example, liposomes, exosomes, or viruses, at solid-liquid interfaces sheds light on their physicochemical and mechanical properties, which has long motivated the development of surface-sensitive measurement techniques. ${ }^{1-3}$ For instance, the adsorption-induced deformation of spherical lipid bilayer nanoparticles, or so-called liposomes, is governed by the balance between the liposomesurface interaction strength, the osmotic pressure difference over the liposomal bilayer, and a material property that is referred to as the membrane bending energy $\kappa^{4-7}$ The magnitude of $\kappa$ depends on the phase state of the membrane. Fluid-phase liposomes have a relatively small $\kappa$ and undergo strong deformation upon adsorption, while gel-phase liposomes have a larger $\kappa$ and undergo weak deformation under equivalent conditions; see, for example, ref 8 . In addition, for sub-100 nm liposomes, $\kappa$ increases as the radius of curvature approaches the membrane thickness; see, for example, ref 9. This curvature effect is important in biological processes such as adhesion, budding and membrane (hemi-) fusion ${ }^{10}$ and motivates the present study into characterizing the deformation of adsorbed liposomes.
To this end, the quartz crystal microbalance (QCM) has proven to be a versatile measurement technique for probing the mass and viscoelastic properties of thin film adsorbates at solid-liquid interfaces. ${ }^{11-13}$ The QCM transducing element is a thin, AT-cut quartz crystal, that is piezoelectrically driven to execute oscillations in shear-thickness mode at the quartz resonance frequency and odd overtones thereof. ${ }^{14,15}$ In conventional embodiments, QCM measures changes in the (overtone) resonance frequency and resonance peak width, or equivalently energy dissipation, due to adsorption events occurring at the sensor surface. ${ }^{16,17}$ The technique is particularly useful for qualitatively distinguishing between different configurations of adsorbed molecules, ${ }^{18}$, for example, phospholipid molecules in liposomes or in supported lipid bilayers. $^{19}$

However, quantitative characterization of adsorbed particles using QCM is complicated by the coupling between adsorbed particles and surrounding fluid. This coupling gives rise to a

Received: August 8, 2017

Accepted: September 21, 2017

Published: September 21, 2017 


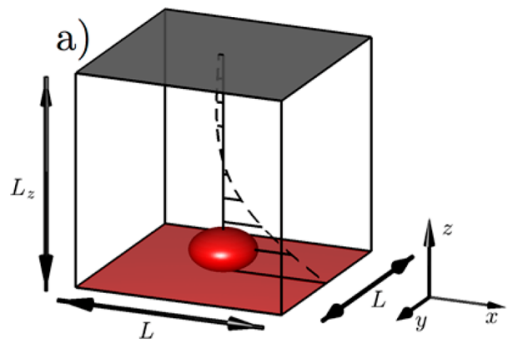

b)

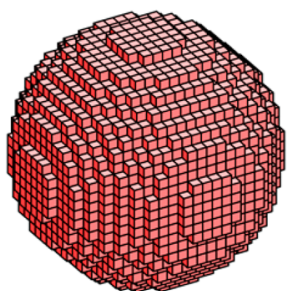

c)

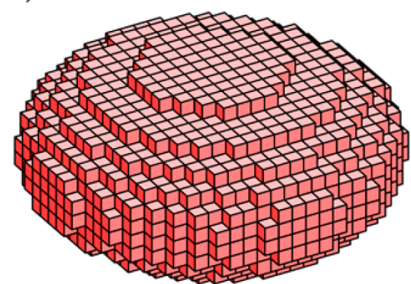

Figure 1. (a) Computational domain of the Lattice Boltzmann (LB) simulation. (b) Staircase approximation of a spherical particle in the LB simulation, where $a_{1} / \Delta x=a_{2} / \Delta x=10$. (c) Staircase approximation of an ellipsoidal particle in the LB simulation, where $a_{1} / \Delta x=12, a_{2} / \Delta x=6$ (aspect ratio $r=a_{1} / a_{2}=2$ ). Here, $a_{1}, a_{2}$, and $\Delta x$ are the particle semimajor axis, semiminor axis, and grid spacing, respectively.

poorly understood, nonlinear relation between the QCM measurement response and the surface coverage of adsorbed particles. $^{20-24}$ To date, most QCM studies did not consider these effects, and instead modeled the adsorbed particles as a homogeneous layer. In this approximation, the particle size can be estimated by the thickness of this layer, based on the Sauerbrey model for rigid films ${ }^{25}$ or the Voigt-Voinova model for viscoelastic films. ${ }^{19,26,27}$ Due to the ignored hydrodynamics, the homogeneous film approximation provides (at most) a qualitative measure of particle deformation. ${ }^{28}$ In this regard, it is noteworthy that an improved homogeneous film approximation has been proposed, one which extrapolates the QCM data to the limit of a zero dissipation shift. ${ }^{29}$ At this point, the equivalent layer behaves as a rigid film, which validates the use of the Sauerbrey relation to estimate the particle size as the thickness of the equivalent layer. ${ }^{5,29-31}$ Reviakine et al. used this extrapolation method to estimate the deformation of adsorbed liposomes. ${ }^{5}$ Despite its improved accuracy, the extrapolation method lacks a rigorous theory for relating the equivalent film height to the particle height, and the method typically underestimates the known particle height by $\sim 20 \%{ }^{13}$ Developing such a theory requires solving the three-dimensional hydrodynamics around adsorbed particles at high surface coverage. Since this is a complex problem to tackle, analytically or numerically, characterizing the shape of adsorbed particles by QCM measurement remains an outstanding challenge.

One promising approach to circumvent the hydrodynamic coupling between particles is to analyze QCM data in the low surface coverage regime. This approach is in marked contrast to the conventional QCM models as described above, which analyze data at high surface coverage. Following this approach, we recently developed a phenomenological model that is based on hydrodynamic simulations and dimensional analysis. ${ }^{32}$ This model provides the particle size and adsorption kinetics from the overtone-dependent QCM frequency shift. Since the model is restricted to nondeformable, spherical particles, we herein extend this model to nonspherical particles, enabling us to analyze deformable, soft-matter particles. To this end, hydrodynamic simulations of an ellipsoid on an oscillating surface are conducted and a model is fitted to the simulation data. The model relates the QCM frequency shift to the size, shape, and surface coverage of the adsorbed particles. To test the model, QCM experiments are performed to measure the adsorption kinetics of fluid-phase and gel-phase liposomes onto a titanium oxide-coated surface. The model is used to determine the corresponding deformation of adsorbed liposomes. By varying the osmotic pressure difference across the liposomal bilayer, we identify, for the different membrane phase states, the respective transition point $\Delta c^{*}$ at which the osmotic pressure-induced liposome deformation becomes appreciable. For the gel-phase liposomes, we also determine the membrane bending energy based on $\Delta c^{*}$.

\section{MATERIALS AND METHODS}

Dimensional Analysis. We consider the QCM frequency shift that arises from $N$ adsorbed particles, in this case liposomes. The particles are deformed and modeled as ellipsoids. We assume that the particles have the same size, the particle shapes do not fluctuate, and the particles are rigidly adhered to the substrate. We also ignore internal fluid motion and the fluidity of the liposomal bilayer. We focus on instances, where there is low surface coverage, that is, when there is a large interparticle spacing such that the hydrodynamic coupling between the adsorbed particles may be neglected, and the QCM frequency shift equals $N$ times the frequency shift of an individual adsorbed particle. The semimajor axis and semiminor axis of the ellipsoids are denoted $a_{1}$ and $a_{2}$, respectively. Based on the above assumptions, the QCM frequency shift $\Delta f$ can be expressed as follows: ${ }^{12,13}$

$$
-\frac{\Delta f}{n}=\frac{N f_{0} \Delta F}{\omega U m_{\mathrm{Q}}}
$$

where $\omega=2 \pi f_{0} n$ and $f_{0}$ are the angular and fundamental QCM frequency, $n$ is the overtone number, $U$ is the QCM surface velocity amplitude, and for ideal resonators $m_{\mathrm{Q}}$ is the mass of the quartz, while for nonideal resonators $m_{\mathrm{Q}}$ is slightly different. $^{13}$ The so-called QCM force $\Delta F$ is the force component, which is in phase with the acceleration of the quartz surface, and which is exerted on the QCM sensor surface due to the adsorption of one ellipsoid. This QCM force $\Delta F=$ $\Delta F_{\mathrm{H}}+m_{\mathrm{P}} \omega U$ is the sum of the hydrodynamic force $\Delta F_{\mathrm{H}}$ and the inertial force of the particle $m_{\mathrm{P}} \omega U$, where $m_{\mathrm{P}}=\rho_{\mathrm{F}}(4 \pi / 3)$ $a_{1}^{2} a_{2}$ is the mass of the particle. It is assumed that the particle mass density equals the fluid mass density $\rho_{\mathrm{F}}$, which is valid for solvent-filled liposomes, as is assumed here, while any known particle mass density can also be taken into account depending on the experimental system. The resulting hydrodynamic force $\Delta F_{\mathrm{H}}$ is the force acting on the combined system of the ellipsoid and the QCM surface minus the force acting on the QCM surface without the ellipsoid.

In Supporting Information, section S1, we use dimensional analysis to derive the following, general form of the relation between $\Delta F_{\mathrm{H}}$ and the dependent variables, which are the velocity amplitude $U$, the angular frequency $\omega$, the particle semi-axes $a_{1}$ and $a_{2}$, the fluid kinematic viscosity $\nu_{\mathrm{F}}$ and the fluid mass density $\rho_{\mathrm{F}}$ : 


$$
\Delta F_{\mathrm{H}}=\rho_{F} a^{3} \omega U \varphi\left(\frac{\delta}{a}, r\right)
$$

Here, $r=a_{1} / a_{2}$ is the aspect ratio of the ellipsoid and the particle radius $a=(A / 4 \pi)^{1 / 2}$ is defined based on the ellipsoid area $A$, which is assumed to be constant under particle deformation.

$$
A=2 \pi a_{1}^{2}\left[1+\left(s^{-1}-s\right) \operatorname{arctanh}(s)\right], \text { with } s^{2}=1-\left(\frac{a_{2}}{a_{1}}\right)^{2}
$$

The viscous penetration depth $\delta=\left(2 \nu_{\mathrm{F}} / \omega\right)^{1 / 2}$, defines the width of the fluid region that is hydrodynamically coupled to an oscillating surface. Within this coupled region, the fluid velocity amplitude decreases exponentially from the value at the surface to zero, with a decay length equal to $\delta$. The corresponding fluid velocity profile is schematically drawn in Figure 1a.

Numerical Simulation. The Lattice Boltzmann (LB) method was employed in order to compute the hydrodynamic force, $\Delta F_{\mathrm{H}}$, which acts on an ellipsoid that is attached to an oscillating surface. The corresponding geometry and coordinate system are presented in Figure 1a. The surface with normal in the $z$ direction oscillates in the $x$ direction, while the $y$ direction is normal to $x$ and $z$. The specifics of the LB method used in this work are described in ref 32 and in Supporting Information, section S2. The method assumes a threedimensional staircase particle shape, as shown in Figure 1b,c. The influence of the staircase approximation on the accuracy of the simulation, as well as that of the sizes of the computational domain, have been verified in Supporting Information, section S2 of ref 32 . Figure 2 visualizes the simulated $x$-component of the fluid velocity in the $(x, z)$-plane for particles of different shapes.

QCM Experiments. We conducted QCM experiments in order to measure the deformation of sub-100 nm, unilamellar phospholipid liposomes that adsorbed onto titanium oxide
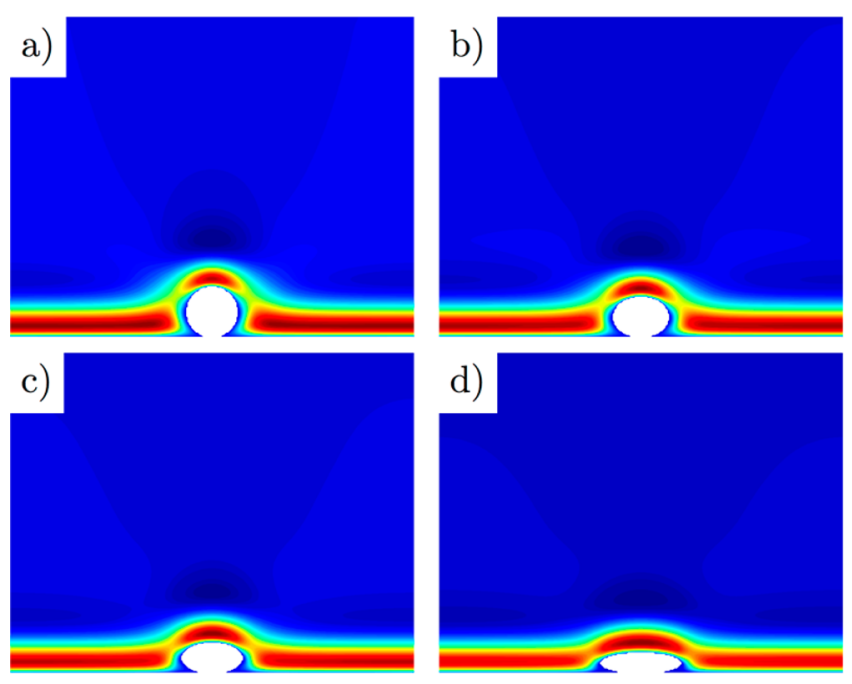

Figure 2. Visualization of the $x$-component of the simulated fluid velocity field around an ellipsoid on the QCM sensor surface, with an aspect ratio (a) $r=1$, (b) $r=1.375$, (c) $r=2$, and (d) $r=4$. Red and blue correspond to maximum and zero velocity, respectively. For all cases, the penetration depth $\delta$ scaled by the particle radius $a$ equals $\delta / a$ $\approx 0.6$, and the snapshot is taken at the moment in oscillation when the surface velocity equals zero.
$\left(\mathrm{TiO}_{2}\right)$ supports, upon which zwitterionic liposomes are known to adsorb but not rupture. ${ }^{33}$ Liposomes were formed by hydrating a dried film of either 1,2-dioleoyl-sn-glycero-3phosphocholine (DOPC; fluid-phase at room temperature) or 1,2-dipalmitoyl-sn-glycero-3-phosphocholine (DPPC; gel-phase at room temperature) (Avanti Polar Lipids, Alabaster, $\mathrm{AL}$ ) in aqueous buffer solution (10 mM Tris $[\mathrm{pH} 7.5]$ with $150 \mathrm{mM}$ $\mathrm{NaCl}$ ) at a lipid concentration of $5 \mathrm{mg} / \mathrm{mL}$, using Milli-Qtreated water with a minimum resistivity of $18.2 \mathrm{M} \Omega \cdot \mathrm{cm}$ (Millipore, Billerica, MA). After vortexing the sample, the liposomes were extruded through a track-etched polycarbonate membrane with a $50 \mathrm{~nm}$ nominal pore diameter. The nominal (intensity-weighted) vesicle radius, measured by dynamic light scattering (DLS, Brookhaven Instrument Co., New York, U.S.A.), was $a=34 \mathrm{~nm}$ for both the fluid- and gel-phase liposomes. Immediately before the start of the QCM experiment, the liposomes were diluted to $5 \mu \mathrm{g} / \mathrm{mL}$ in a 10 $\mathrm{mM}$ Tris buffer ( $\mathrm{pH} 7.5$ ) with varying $\mathrm{NaCl}$ concentration. To modulate the extent of liposome deformation, the external ionic strength $c_{\text {out }}=\left[\mathrm{Na}^{+}\right]=\left[\mathrm{Cl}^{-}\right]$was varied between $c_{\text {out }}=100$ and $500 \mathrm{mM}$, while the internal ionic strength was kept fixed at $c_{\text {in }}=$ $150 \mathrm{mM}$. Since the time scale of $\mathrm{Na}^{+}$and $\mathrm{Cl}^{-}$diffusion across lipid bilayers is appreciably longer than the experimental time scale, $^{34}$ the liposomes in bulk solution are subjected to a positive $\left(c_{\text {out }}>150 \mathrm{mM}\right)$, negative $\left(c_{\text {out }}<150 \mathrm{mM}\right)$, or zero $\left(c_{\text {out }}=150 \mathrm{mM}\right)$ osmotic pressure, and the corresponding conditions are referred to as hypertonic, hypotonic, and isotonic, respectively. In addition to the osmotic pressure, the ionic strength also affects the surface adhesion force as discussed below, see, for example, ref 35 .

Liposome adsorption was measured by the QCM technique using the Q-Sense E4 instrument (Biolin Scientific AB, Stockholm, Sweden) with a fundamental frequency of $f_{0}=5$ $\mathrm{MHz}$ and the sensor surface had a sputter-coated, $50 \mathrm{~nm}$ thick layer of titanium oxide (model No. QSX 310, Biolin Scientific $A B$ ). A peristaltic pump (Reglo Digital, Ismatec, Glattbrugg, Switzerland) was used to inject liquid samples into the QCM measurement chamber at a flow rate of $50 \mu \mathrm{L} / \mathrm{min}$. The temperature in the measurement chamber was maintained at $25.0 \pm 0.5{ }^{\circ} \mathrm{C}$. The experimental data were collected at the first to 13th odd overtones using the QSoft software program (Biolin Scientific $\mathrm{AB}$ ). Baseline signals in aqueous buffer solution were recorded for $7 \mathrm{~min}$ prior to liposome injection.

Shape Calculations. The shape of a suspended liposome depends on the osmotic pressure difference across the liposomal bilayer. In bulk solution, hypotonic $\left(c_{\text {out }}<c_{\text {in }}\right)$ or isotonic $\left(c_{\text {out }}=c_{\text {in }}\right)$ liposomes are spherical while hypertonic $\left(c_{\text {out }}>c_{\text {in }}\right)$ liposomes may be deformed depending on the membrane bending energy. When a liposome is adsorbed onto a solid surface, the liposome-substrate adhesion force tends to induce a greater degree of liposome deformation and the extent of deformation depends on the balance between osmotic pressure, surface adhesion energy, and bending energy. The shape can be calculated by minimizing the following energy $E$ functional. ${ }^{4}$

$$
E=w A_{\mathrm{w}}+\sigma A+\Delta p V+\frac{\kappa}{2} \oint H^{2} \mathrm{~d} A
$$

Here, $w$ is the surface adhesion energy density, $A_{\mathrm{w}}$ is the contact area between the deformed liposome and the solid substrate, $\sigma$ is the membrane surface tension, $A$ is the membrane area, $\Delta p$ is the difference between the external and the internal osmotic pressure (in the deformed state), $V$ is the volume of the 

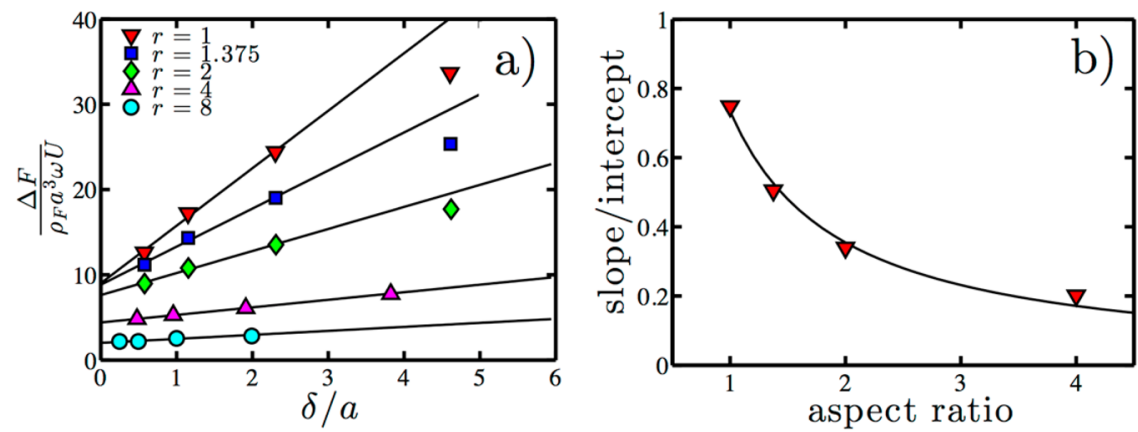

Figure 3. (a) Simulated QCM force per particle $\Delta F$ vs scaled penetration depth $\delta / a$ for various aspect ratios $r$. The particle radius is defined as $a=$ $(A / 4 \pi)^{1 / 2}$, where $A$ is the particle area, $U$ is the QCM surface velocity amplitude, $\omega$ is the QCM angular frequency, and $\rho_{\mathrm{F}}$ is the fluid mass density. The numerical error of the computed force was estimated in ref 32 and found to be smaller than the marker size. (b) QCM shape signature $S / I$ as a function of $r$, where $S$ and $I$ are the slope and intercept of instantaneous QCM data on $(\delta / a,-\Delta f / n)$-coordinates. The markers are the simulated values and the line corresponds to eq 10 .

deformed liposome, $\kappa$ is the membrane bending energy, and $H$ is the mean membrane curvature. We numerically solve the axisymmetric membrane shape by minimizing eq 4 using the method of ref 36 , which is extended to take into account surface adhesion using the boundary conditions outlined in ref 37. The calculations utilize $\kappa, w, a$, and $V$ as input parameters and generate the liposome shape, $A_{\mathrm{w}}, \sigma$, and $\Delta p$ as output parameters, where the latter two act as Lagrange multipliers in order to satisfy the liposome area and volume constraints. The one-dimensional (axisymmetric) shape equation is discretized using $2 \times 10^{4}$ grid points equidistantly distributed over the meridian and it was numerically solved using the six-stage, fifthorder Runge-Kutta method of MATLAB (Ode45). The aspect ratio $r$ is formally defined as the ratio of the maximum height and width of the computed shape. The external ionic strength $c_{\text {out }}$ is related to the osmotic pressure difference in the deformed state $\Delta p$, the relative deformed liposome volume $V$ / $V_{0}$, and the ionic strength in the nondeformed liposome $c_{\text {in }}$ by

$$
c_{\text {out }}=\frac{\Delta p}{2 R T}+c_{\text {in }} \frac{V_{0}}{2 V}
$$

where $R$ is the universal gas constant, $T$ is the absolute temperature, $V_{0}=4 a^{3} \pi / 3$ is the volume of the nondeformed liposome, the liposome radius $a=(A / 4 \pi)^{1 / 2}$ is defined based on the liposome surface area $A$, and the factors of two reflect that the total number of ions per volume is twice the ionic strength, $c_{\text {in }}$ or $c_{\text {out }}$ in a monovalent salt solution $(\mathrm{NaCl})$.

In addition to general shapes, we also analyze the limiting case of a negligible surface adhesion force and an infinitesimal deformation. In this case, the liposome shape can be assumed to be ellipsoidal. In Supporting Information, section S3, we derive that, in this case, the liposome shape deforms when the ionic strength difference $c_{\text {out }}-c_{\text {in }}$ exceeds a critical value $\Delta c^{*}$ of

$$
\Delta c^{*}=\frac{8 \pi \kappa}{R T V_{0}}
$$

Equation 6 indicates that a greater positive osmotic pressure is required to deform a liposome with a greater membrane bending energy.

\section{RESULTS}

Hydrodynamic Simulation. Guided by dimensional analysis (eq 2), we seek an expression for the scaled QCM force $\Delta F / \rho_{\mathrm{F}} a^{3} \omega U$ as a function of the particle aspect ratio $r$ and the scaled viscous penetration depth $\delta / a$. Here, $\Delta F=m_{\mathrm{P}} \omega U+$
$\Delta F_{\mathrm{H}}$ is the sum of the particle inertia $m_{\mathrm{p}} \omega U$ and the hydrodynamic force component $\Delta F_{\mathrm{H}}$, that is in phase with the quartz acceleration, that is, which contributes to the QCM frequency shift. For this purpose, we have conducted simulations for various $r$, ranging between 1 and 8 , and for various $\delta / a$, ranging between 0.6 and 4.6 ; see Supporting Information, Table $S 1$. The simulation results in Figure 3a show that $\Delta F / \rho_{\mathrm{F}} a^{3} \omega U$ increases as a function of $\delta / a$, which reflects a stronger fluid coupling with decreasing frequency, while $\Delta F / \rho_{\mathrm{F}} a^{3} \omega U$ decreases as a function of $r$, which reflects a weaker fluid coupling with decreasing particle volume.

For each of the considered $r$, we find that $\Delta F / \rho_{\mathrm{F}} a^{3} \omega U$ is a linear function of $\delta / a$ roughly up to $\delta / a=3$, while beyond this value, $\Delta F / \rho_{\mathrm{F}} a^{3} \omega U$ deviates from linearity. Noting that, for the current experimental setup, $\delta / a<3$ is satisfied for liposomes with $a>30 \mathrm{~nm}$ and $n>3$, we focus on the linear regime and approximate the data in Figure $3 a$ using a first-order Taylor expansion in $\delta / a$ :

$$
\frac{\Delta F}{\rho_{\mathrm{F}} a^{3} \omega U}=\alpha_{0}(r)+\alpha_{1}(r) \frac{\delta}{a}
$$

where the expansion coefficients $\alpha_{0}$ and $\alpha_{1}$ are functions of $r$ and it is noted that the particle inertia is absorbed into the $\alpha_{0}$ term. Combining eqs 1 and 7 yields

$$
\frac{-\Delta f}{n}=\frac{N f_{0} \rho_{\mathrm{F}} a^{3}}{m_{\mathrm{Q}}}\left[\alpha_{0}(r)+\alpha_{1}(r) \frac{\delta}{a}\right]=I+S \frac{\delta}{a}
$$

which shows that $\alpha_{1}$ and $\alpha_{0}$ are proportional to the slope $S$ and the intercept $I$ of the QCM measurement data on $(\delta / a,-\Delta f / n)$ coordinates:

$$
S=\frac{\partial}{\partial(\delta / a)}\left(\frac{-\Delta f}{n}\right)_{\delta / a=0}, I=\left(\frac{-\Delta f}{n}\right)_{\delta / a=0}
$$

The ratio $S / I=\alpha_{1} / \alpha_{0}$ is referred to as the QCM shape signature, and Figure $3 \mathrm{~b}$ shows the corresponding simulation data as a function of $r$. Parameterizing these data using

$$
\frac{S}{I}=0.73 r^{-1.05}
$$

results in the following expression for the particle aspect ratio:

$$
r=0.74\left[\frac{S}{I}\right]^{-0.95}
$$



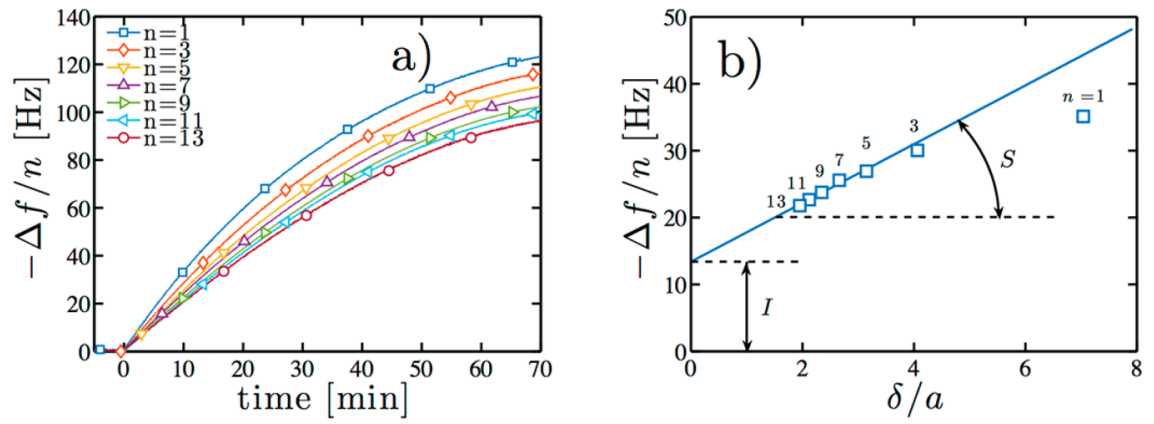

Figure 4. (a) Measured frequency shift $-\Delta f / n$ for fluid-phase DOPC liposomes in $c_{\text {out }}=150 \mathrm{mM}$ ionic strength for the nearly complete adsorption spectrum. (b) Experimental data at $t=10 \mathrm{~min}$ on $(\delta / a,-\Delta f / n)$ coordinates. Particle aspect ratio can be determined from the slope $S$ and intercept $I$ using eq 11 . The measurement noise is smaller than the marker size.
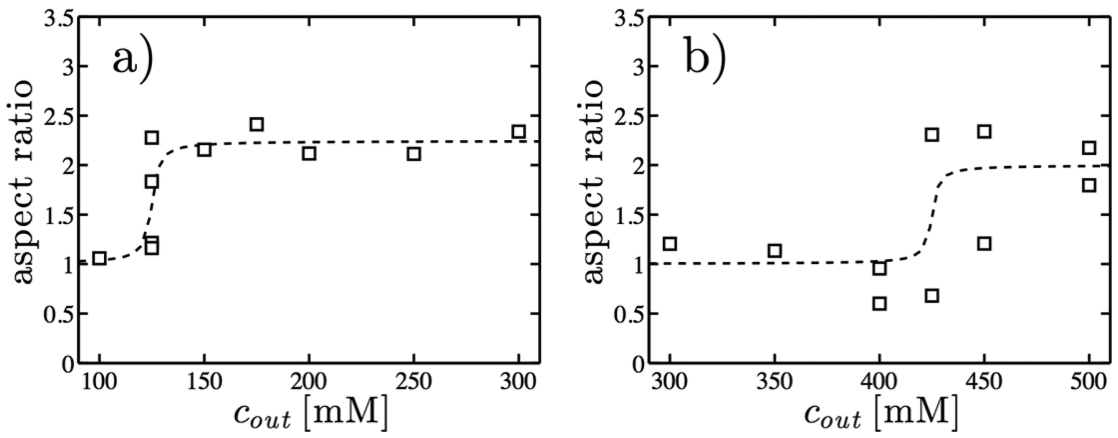

Figure 5. Measured liposome aspect ratio $r$ as a function of external ionic strength $c_{\text {out }}$ for (a) fluid-phase DOPC liposomes and (b) gel-phase DPPC liposomes. Each data point represents the result obtained from an independent experiment.

Next, we use eq 11 to extract the extent of deformation of liposomes from experimental QCM data.

Liposome Adsorption Experiments. Figure 4a presents the QCM frequency shift $-\Delta f / n$ as a function of time for overtones $n=1-13$ due to the adsorption of fluid-phase DOPC liposomes in isotonic conditions onto a $\mathrm{TiO}_{2}$ surface. Our model to extract particle shape from QCM data (eq 11) assumes a low surface coverage of particles, that is, a large interparticle distance such that there is negligible hydrodynamic coupling between adsorbed particles. Therefore, we focus on relatively short times after starting liposome injection, when the absence of hydrodynamic coupling is reflected in the linear frequency shift response as a function of time. Figure 4a shows that this initial linear response in the low coverage regime extends up to $t \sim 10 \mathrm{~min}$, which corresponds to $-\Delta f / n \sim 25$ $\mathrm{Hz}$ for $n=3$. In ref 32, we have estimated that, for $a \sim 4 \times 10^{-8}$ $\mathrm{m}$ and $-\Delta f / n \sim 25 \mathrm{~Hz}$, there is an average interparticle distance of $\sim 20$ particle radii, which is consistent with a low surface coverage of particles in this regime.

Applying eq 11 to extract particle shape requires evaluating the instantaneous $-\Delta f / n$ as a function of the scaled penetration depth $\delta / a$ in the linear, low coverage regime, which is illustrated in Figure $4 \mathrm{~b}$ for $t=10 \mathrm{~min}$. Here, the markers correspond to the various overtones. Similar to the simulation data in Figure 3a, the experimental data in Figure $4 \mathrm{~b}$ follow a straight line for $n>3$, which corresponds to $\delta / a<3$. The first and third overtones deviate from this linearity, which is consistent with the deviation from linearity for $\delta / a>3$ that is observed in the simulation data. As our model (eq 11) is based on a linear fit to the data on the $(\delta / a,-\Delta f / n)$ plane, we focus the subsequent analysis on $n>3$. The intercept $I$ and the slope $S$ of this fit (cf. Figure $4 \mathrm{~b}$ ) are inserted in eq 11, which gives us a liposome aspect ratio of $r \approx 2.2$. This suggests that, for this case, the fluid-phase DOPC liposomes in isotonic conditions are deformed due to the surface adhesion force. It is noted that this result is independent of $t$ because, in the low coverage regime, $S$ and $I$ are linear functions of $t$ and, hence, the argument $S / I$ of eq 11 is independent of time. Following this measurement approach, we next investigated the effect of osmotic pressure on the aspect ratio $r$ of adsorbed DOPC liposomes, where we varied the external ionic strengths $c_{\text {out }}$ between 100 and $300 \mathrm{mM}$, while keeping the internal ionic strength fixed at $c_{\text {in }}=150 \mathrm{mM}$. The results in Figure 5a show $r$ $\approx 1.0$ for $c_{\text {out }}<c_{\text {in }}$ and $r \approx 2.2$ for $c_{\text {out }} \geq c_{\text {in }}$.

The critical ionic strength difference at the onset of membrane deformation was determined to be $\Delta c^{*}=c_{\text {out }}-$ $c_{\text {in }} \approx-25 \mathrm{mM}$, by fitting

$$
r=1+\frac{r_{\max }-1}{2}\left[\frac{2}{\pi} \arctan \left(\frac{\Delta c-\Delta c^{*}}{\delta \Delta c}\right)+1\right]
$$

to the experimental data in Figure 5a, where $r_{\max }, \Delta c^{*}$, and $\delta \Delta c$ are fitting parameters. As shown in Figure $5 a, b$, measurements were repeated under identical experimental conditions and there was only a minor variation in the resulting $r$ for conditions away from the transition point $\left(\Delta c-\Delta c^{*} \gtrsim 50\right.$ $\mathrm{mM})$, while there were larger variations around the transition point for $\Delta c-\Delta c^{*} \lesssim 50 \mathrm{mM}$. These larger variations most likely reflect the sensitivity of liposome deformation to small differences in osmotic pressure conditions around the transition point.

It is further observed that $r$ does not change upon increasing $\Delta c$ beyond $\Delta c^{*}$, but instead saturates at $r \approx 2.2$. This saturation may reflect inhibition of membrane bending when the radius of the membrane curvature approaches the membrane thickness; see, for example, ref 9 . The fact that we obtain the expected 

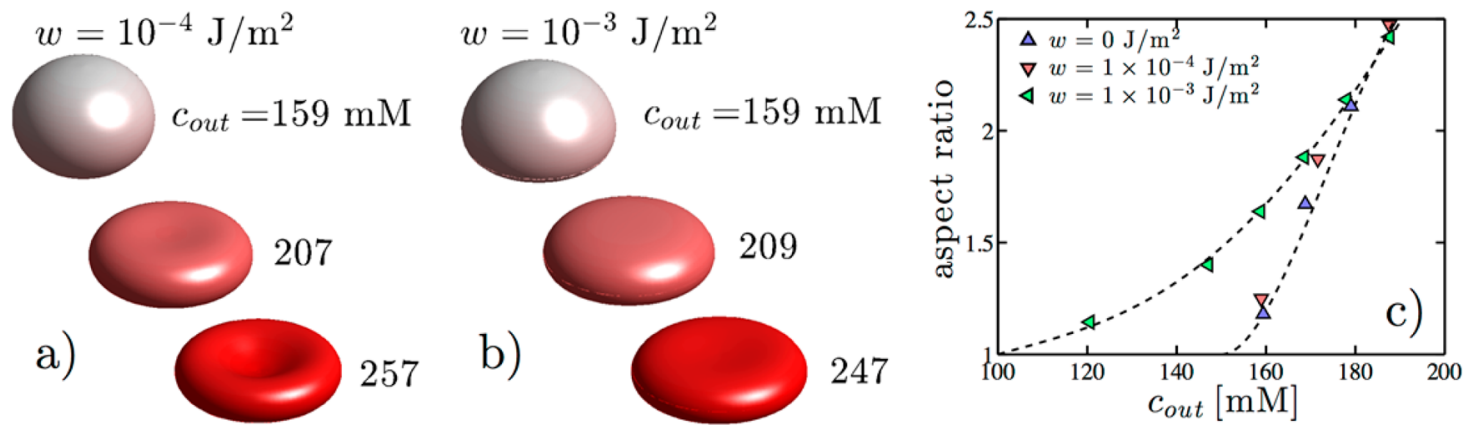

Figure 6. Theoretical shapes of fluid-phase liposomes (computed with eq 4) as functions of external ionic strength $c_{\text {out }}$ for a relatively (a) small or (b) large surface adhesion energy $w a^{2} / \kappa=1$ or 10, respectively. (c) Theoretical liposome aspect ratio $r$ as a function of the external ionic strength $c_{\text {out }}$ for various surface adhesion energies, $w$, for fluid-phase liposomes. The calculations are based on a liposome radius of $a=34 \mathrm{~nm}$, a membrane bending energy of $\kappa=30 k_{\mathrm{B}} T$, and an internal ionic strength of $c_{\text {in }}=150 \mathrm{mM}$. The dashed lines are drawn to guide the eye.

result of $r \approx 1.0$ for $\Delta c<\Delta c^{*}$ supports that the model captures the essential physics and that in the current experiments the liposomes behave in reasonable agreement with the simulated conditions, that is, there are negligible shape fluctuations and negligible rocking, sliding, or rolling motions.

In addition to fluid-phase DOPC liposomes, we also investigated the aspect ratio $r$ of adsorbed, gel-phase DPPC liposomes as a function of the external ionic strength $c_{\text {out }}$ which was varied between 300 and $500 \mathrm{mM}$, while keeping the internal ionic strength fixed at $c_{\text {in }}=150 \mathrm{mM}$. Figure $5 \mathrm{~b}$ shows the resulting $r$ as a function of $c_{\text {out }}$. For the gel-phase liposomes, deformation sets in beyond a critical ionic strength difference of $\Delta c^{*}=c_{\text {out }}-c_{\text {in }} \approx 275 \mathrm{mM}$. The larger critical ionic strength difference observed for the DPPC liposomes, as compared to the DOPC liposomes, supports that a larger osmotic pressure force is needed to deform liposomes with a larger membrane bending energy.

Calculation of Membrane Bending Energy. Figure 5a shows that fluid-phase liposomes deform beyond a critical ionic strength of $\Delta c^{*} \approx-25 \mathrm{mM}$. This value represents the (negative) osmotic pressure that is needed to counteract the surface adhesion force $w$ and keep the liposomes spherical. To estimate the corresponding value for $w$, we theoretically analyze the shapes of deformed liposomes by minimizing the energy functional (eq 4). We compute the shapes of adsorbed liposomes with a radius of $a=34 \mathrm{~nm}$, a bending energy of $\kappa$ $=30 k_{\mathrm{B}} T,{ }^{8,9}$ and consider two values for the surface adhesion force: $w=10^{-4}$ and $10^{-3} \mathrm{~J} \mathrm{~m}^{-2}$, which are in the range of experimentally measured forces between lipid bilayers and oxide surfaces. ${ }^{35,38}$ Figure $6 \mathrm{a}, \mathrm{b}$ shows the computed shapes and Figure $6 c$ shows the corresponding aspect ratio values for various external ionic strength conditions $c_{\text {out }}$, while keeping the internal ionic strength fixed at $c_{\text {in }}=150 \mathrm{mM}$. For $w=10^{-4} \mathrm{~J}$ $\mathrm{m}^{-2}\left(w a^{2} / \kappa=1\right)$, there is a negligible adhesion effect on liposome deformation, which sets in at $\Delta c^{*}=c_{\text {out }}-c_{\text {in }} \approx 0$ $\mathrm{mM}$. On the other hand, for $w=10^{-3} \mathrm{~J} \mathrm{~m}^{-2}\left(w a^{2} / \kappa=10\right)$, there is a noticeable adhesion effect and deformation sets in at a smaller $\Delta c^{*} \approx-50 \mathrm{mM}$. Experimentally, the deformation of fluid-phase liposomes sets in at $\Delta c^{*} \approx-25 \mathrm{mM}$ (cf. Figure 5a), suggesting that, in the present system, the surface interaction strength lies in the range $10^{-4} \mathrm{~J} \mathrm{~m}^{-2}<w<10^{-3} \mathrm{~J} \mathrm{~m}^{-2}$, that is, 1 $<a^{2} w / \kappa<10$, which is within the range reported in refs 35 and 38 .

It is emphasized that our modeling approach is based on the assumption of an ellipsoidal particle shape, which is in reasonable agreement with the computed shapes in Figure 6a,b. For larger particles and for particles with a smaller bending energy, the adsorbed shape can deviate from ellipsoidal to become more like a spherical cap. ${ }^{4}$ For the present case, the corresponding differences in hydrodynamic coupling are expected to be minor $(<10 \%)$ and do not affect the estimation of the transition point, which is independent of the details of the particle shape.

Next, we use eq 6 to relate the bending energy $\kappa$ of adsorbed liposomes to the critical ionic strength difference $\Delta c^{*}=c_{\text {out }}-c_{\text {in }}$ at the onset of liposome deformation. This equation is based on the assumption of a negligible surface adhesion force, i.e., a small $a^{2} w / \kappa$. We estimated above that, for fluid-phase DOPC liposomes, $1<a^{2} w / \kappa<10$, which suggests that the surface adhesion energy plays an important role. Furthermore, the measured $\Delta c^{*} \approx-25 \mathrm{mM}$ (cf. Figure 5a) is on the order of the smallest ionic strength difference that could be prepared with suitable accuracy. These considerations invalidate the use of eq 6 to determine $\kappa$ for the fluid-phase liposomes. Importantly, on the other hand, for the gel-phase DPPC liposomes, $\Delta c^{*} \approx 275$ $\mathrm{mM}$ (cf. Figure $5 \mathrm{~b}$ ) and the corresponding, critical osmotic pressure is an order of magnitude larger than the (negative) osmotic pressure equivalent of the surface adhesion force $(-25$ $\mathrm{mM}$; cf. Figure 5a). This implies that, at the transition point, the surface adhesion force has a minor effect, which validates the use of eq 6 to compute the corresponding bending energy. This procedure results in $\kappa=1080 k_{\mathrm{B}} T$, which is $2-4$ times as large as the typically measured values for DPPC and other gelphase lipid compositions, that is, $\kappa=300-600 k_{\mathrm{B}} T .^{39-41}$ The large $\kappa$ is likely related to the high membrane curvature of the small liposomes used in the present study. Indeed, while the quoted values for $\kappa$ are measured for liposomes of $\sim 10 \mu \mathrm{m}$ diameter, larger values for $\kappa$ have been reported for liposome diameters below $\sim 200 \mathrm{~nm} .^{5,9,28,42}$ In this respect, it is noteworthy that a similarly large value for $\kappa$ was found in a related QCM study on gel-phase liposomes of similar size. ${ }^{5}$ Hence, the QCM model applied herein provides a method to determine the aspect ratio and bending energy of adsorbed liposomes, and the value obtained is consistent with the literature.

\section{CONCLUSIONS}

Based on dimensional analysis and numerical simulation of the hydrodynamics of an ellipsoid attached to an oscillating surface, we have derived a model (eq 11) that relates the aspect ratio $r$ of absorbed particles to the slope and intercept of the line that fits instantaneous, overtone-dependent QCM data on $(\delta / a$, 
$-\Delta f / n)$ coordinates. Here, $\delta$ is the viscous penetration depth, $a$ is the particle radius, $\Delta f$ is the QCM frequency shift, and $n$ is the overtone number. The model concerns the initial adsorption phase, where there are negligible hydrodynamic interactions between the adsorbed particles.

We have applied the model to QCM data pertaining to the adsorption of liposomes with a nominal intensity-weighted radius of $a=34 \mathrm{~nm}$ and with different lipid compositions, that are in either the fluid-phase or gel-phase at room temperature. The conformation of the liposomes was changed by varying the ionic $(\mathrm{NaCl})$ strength in the liposome exterior $c_{\text {out }}$, while keeping the ionic strength in the liposome interior $c_{\text {in }}$ fixed. For fluid-phase liposomes under hypotonic conditions $\left(c_{\text {out }}-c_{\text {in }} \lesssim\right.$ $-25 \mathrm{mM}$ ), the method reveals that the adsorbed liposomes remain spherical such that $r \approx 1$, while under isotonic and hypertonic conditions $\left(c_{\text {out }}-c_{\text {in }} \gtrsim-25 \mathrm{mM}\right)$, the method reveals the deformation of adsorbed liposomes. The results are consistent with a membrane bending model, based on a surface adhesion force in the range of $10^{-4} \mathrm{~J} \mathrm{~m}^{-2}<w<10^{-3} \mathrm{~J} \mathrm{~m}^{-2}$. On the other hand, the stiffer, gel-phase liposomes deform when the ionic strength difference exceeds a critical value of $c_{\text {out }}-c_{\text {in }}$ $\approx 275 \mathrm{mM}$. Using the membrane bending model, we determined that the corresponding value for the bending energy of these gel-phase liposomes is a few-fold larger than previous measurements for $\sim 10 \mu \mathrm{m}$ liposomes, while it is consistent with previous measurements for liposomes with diameters below $\sim 200 \mathrm{~nm}^{5,9,28,42}$

It is interesting to point out the difference between the present method and that of ref 5 to extract particle deformation from QCM data. Specifically, the present method requires the overtone dependence of the instantaneous frequency shift at low surface coverage, whereas the method of ref 5 requires the time dependence of (at least) one overtone of both the frequency and the dissipation shifts at high surface coverage. The coexistence of these two independent pathways to interpret the deformation of adsorbed particles is striking and demonstrates the excellent and broad capabilities of the QCM technique to measure the geometrical properties of heterogeneous adsorbates.

The ability of the proposed model to extract liposome shape illustrates that the geometrical properties of adsorbate particles are encoded within QCM overtone data, and the present simulation methodology provides an analytical framework to evaluate the shape deformation of adsorbed particles. The approach outlined in this work is therefore not restricted to characterizing liposomes, but also applicable to a wide range of small particles such as nanoscopic functional materials. ${ }^{43}$

\section{ASSOCIATED CONTENT}

\section{S Supporting Information}

The Supporting Information is available free of charge on the ACS Publications website at DOI: 10.1021/acs.analchem.7b03179.

Details of the dimensional analysis, Lattice Boltzmann simulation, and analysis of shape deformation analysis (PDF).

\section{AUTHOR INFORMATION}

\section{Corresponding Author}

*E-mail: njcho@ntu.edu.sg.

ORCID 1

Nam-Joon Cho: 0000-0002-8692-8955

\section{Notes}

The authors declare no competing financial interest.

\section{ACKNOWLEDGMENTS}

This article is dedicated to the memory of Dr. Kay K. Kanazawa, a long-time friend and colleague who provided stimulating advice that motivated work in this direction. We would also like to acknowledge funding support from the National Research Foundation of Singapore (NRF2015NRFPOC001-019) to N.-J.C.

\section{REFERENCES}

(1) Höök, F.; Kasemo, B.; Grunze, M.; Zauscher, S. ACS Nano 2008, 2, 2428-2436.

(2) Mashaghi, A.; Mashaghi, S.; Reviakine, I.; Heeren, R. M.; Sandoghdar, V.; Bonn, M. Chem. Soc. Rev. 2014, 43, 887-900.

(3) Jackman, J. A.; Rahim Ferhan, A.; Cho, N.-J. Chem. Soc. Rev. 2017, 46, 3615-3660.

(4) Lipowsky, R.; Seifert, U. Mol. Cryst. Liq. Cryst. 1991, 202, 17-25.

(5) Reviakine, I.; Gallego, M.; Johannsmann, D.; Tellechea, E. J. Chem. Phys. 2012, 136, 084702.

(6) Jackman, J. A.; Choi, J.-H.; Zhdanov, V. P.; Cho, N.-J. Langmuir 2013, 29, 11375-11384.

(7) Jackman, J. A.; Špačková, B.; Linardy, E.; Kim, M. C.; Yoon, B. K.; Homola, J.; Cho, N.-J. Chem. Commun. 2016, 52, 76-79.

(8) Dimova, R. Adv. Colloid Interface Sci. 2014, 208, 225-234.

(9) Takechi-Haraya, Y.; Sakai-Kato, K.; Abe, Y.; Kawanishi, T.; Okuda, H.; Goda, Y. Langmuir 2016, 32, 6074.

(10) Tabaei, S. R.; Gillissen, J. J. J.; Vafaei, S.; Groves, J. T.; Cho, N.-J. Nanoscale 2016, 8, 13513-13520.

(11) Arnau, A. Piezoelectric Transducers and Applications; Springer, 2004.

(12) Reviakine, I.; Johannsmann, D.; Richter, R. P. Anal. Chem. 2011, $83,8838-8848$.

(13) Johannsmann, D. The Quartz Crystal Microbalance in Soft Matter Research: Fundamentals and Modeling; Springer, 2014.

(14) Reed, C.; Kanazawa, K. K.; Kaufman, J. J. Appl. Phys. 1990, 68, 1993-2001.

(15) Arnau, A. Sensors 2008, 8, 370-411.

(16) Rodahl, M.; Höök, F.; Krozer, A.; Brzezinski, P.; Kasemo, B. Rev. Sci. Instrum. 1995, 66, 3924-3930.

(17) Rodahl, M.; Kasemo, B. Rev. Sci. Instrum. 1996, 67, 3238-3241.

(18) Marx, K. A. Biomacromolecules 2003, 4, 1099-1120.

(19) Keller, C.; Kasemo, B. Biophys. J. 1998, 75, 1397-1402.

(20) Bingen, P.; Wang, G.; Steinmetz, N. F.; Rodahl, M.; Richter, R. P. Anal. Chem. 2008, 80, 8880-8890.

(21) Edvardsson, M.; Svedhem, S.; Wang, G.; Richter, R.; Rodahl, M.; Kasemo, B. Anal. Chem. 2009, 81, 349-361.

(22) Carton, I.; Brisson, A. R.; Richter, R. P. Anal. Chem. 2010, 82, 9275-9281.

(23) Rojas, E.; Gallego, M.; Reviakine, I. Anal. Chem. 2008, 80, $8982-8990$

(24) Johannsmann, D.; Brenner, G. Anal. Chem. 2015, 87, 74767484.

(25) Sauerbrey, G. Eur. Phys. J. A 1959, 155, 206-222.

(26) Voinova, M. V.; Rodahl, M.; Jonson, M.; Kasemo, B. Phys. Scr. 1999, 59, 391.

(27) Johannsmann, D. Macromol. Chem. Phys. 1999, 200, 501-516.

(28) Jackman, J. A.; Yorulmaz Avsar, S.; Ferhan, A. R.; Li, D.; Park, J. H.; Zhdanov, V. P.; Cho, N.-J. Anal. Chem. 2017, 89, 1102-1109.

(29) Tellechea, E.; Johannsmann, D.; Steinmetz, N. F.; Richter, R. P.; Reviakine, I. Langmuir 2009, 25, 5177-5184.

(30) Zhu, T.; Jiang, Z.; Nurlybaeva, E. M. R.; Sheng, J.; Ma, Y. Langmuir 2013, 29, 6377-6385.

(31) Olsson, A. L.; Quevedo, I. R.; He, D.; Basnet, M.; Tufenkji, N. ACS Nano 2013, 7, 7833-7843.

(32) Gillissen, J. J. J.; Tabaei, S. R.; Jackman, J. A.; Cho, N.-J. Analyst 2017, 142, 3370-3379. 
(33) Jackman, J. A.; Zan, G. H.; Zhao, Z.; Cho, N.-J. Langmuir 2014, 30, 5368-5372.

(34) Papahadjopoulos, D. Biochim. Biophys. Acta, Biomembr. 1971, 241, 254-259.

(35) Anderson, T. H.; Min, Y.; Weirich, K. L.; Zeng, H.; Fygenson, D.; Israelachvili, J. N. Langmuir 2009, 25, 6997-7005.

(36) Deuling, H.; Helfrich, W. J. Phys. (Paris) 1976, 37, 1335-1345.

(37) Seifert, U.; Lipowsky, R. Phys. Rev. A: At., Mol., Opt. Phys. 1990, $42,4768$.

(38) Schönherr, H.; Johnson, J. M.; Lenz, P.; Frank, C. W.; Boxer, S. G. Langmuir 2004, 20, 11600-11606.

(39) Dimova, R.; Pouligny, B.; Dietrich, C. Biophys. J. 2000, 79, 340356.

(40) Lee, C.-H.; Lin, W.-C.; Wang, J. Phys. Rev. E: Stat. Phys., Plasmas, Fluids, Relat. Interdiscip. Top. 2001, 64, 020901.

(41) Tristram-Nagle, S.; Liu, Y.; Legleiter, J.; Nagle, J. F. Biophys. J. 2002, 83, 3324-3335.

(42) Jackman, J. A.; Zan, G. H.; Zhdanov, V. P.; Cho, N.-J. J. Phys. Chem. $B$ 2013, 117, 16117-16128.

(43) Ariga, K.; Li, J.; Fei, J.; Ji, Q.; Hill, J. P. Adv. Mater. 2016, 28, 1251-1286. 